PROCEEDINGS OF THE

AMERICAN MATHEMATICAL SOCIETY

Volume 125, Number 9, September 1997, Pages 2657-2660

S 0002-9939(97)03861-6

\title{
A STRICT VERSION OF THE NON-COMMUTATIVE URYSOHN LEMMA
}

\author{
GERT K. PEDERSEN
}

(Communicated by Palle E. T. Jorgensen)

\begin{abstract}
Given a pair $B, C$ of $q$-commuting, hereditary $C^{*}$-subalgebras of a unital $C^{*}$-algebra $A$, such that $B \cap C$ is $\sigma$-unital and $1 \in B+C$, there is an element $h$ in $A$, with $0 \leq h \leq 1$, such that $h$ is strictly positive in $B$ and $1-h$ is strictly positive in in $C$. Moreover, $h-h^{2}$ is strictly positive in in $B \cap C$.
\end{abstract}

As a motivation for Urysohn's Lemma on a normal space $X$ it is often pointed out that if $X$ were a metric space the result would be almost trivial: Given disjoint, closed subsets $E$ and $F$ in $X$ define $f$ in $C(X)$ by

$$
f(x)=\operatorname{dist}(x, F)(\operatorname{dist}(x, F)+\operatorname{dist}(x, E))^{-1} .
$$

This Urysohn function has the added distinction that it lies strictly between 0 and 1 off $E \cup F$. Actually, the existence of such a strict Urysohn function is assured in more general cases: If $X \backslash(E \cup F)$ is a co-zero set, i.e. for some function $b$ in $C(X)$ we have $E \cup F=\{x \in X \mid b(x)=0\}$, then, assuming that $0 \leq b$ and $\|b\|_{\infty}<1$, we choose an arbitrary Urysohn function $g$, relative to $E$ and $F$, and obtain a strict one $f$ by taking

$$
f=g(1-b)+(1-g) b .
$$

In [1], [2] and [3] Akemann showed that there is a perfect generalization of the Urysohn Lemma to the non-commutative setting of $C^{*}$-algebras. His results were subsequently generalized by Brown, who proved the non-commutative version of interpolating a continuous function between a given pair of semi-continuous functions $[5,3.16]$. Brown's argument was adapted to give a short proof of the AkemannUrysohn theorem in $[4,2.7]$.

The aim of this note is to establish a strict version of the Akemann-Urysohn result. In its most palatable formulation it sounds as follows:

Theorem 1. Let $B$ and $C$ be hereditary $C^{*}$-subalgebras of a separable, unital $C^{*}$ algebra $A$ such that $1 \in B+C$. If $B$ and $C$ have approximate units $\left(u_{n}\right)$ and $\left(v_{n}\right)$, respectively, such that $u_{n} v_{n}-v_{n} u_{n}$ converges to zero in the $\sigma\left(A, A^{*}\right)$-topology, then there is an element $h$ in $A$, with $0 \leq h \leq 1$, such that $h$ is strictly positive in $B$ and $1-h$ is strictly positive in $C$. Moreover, $h-h^{2}$ is strictly positive in $B \cap C$.

Received by the editors November 13, 1995 and, in revised form, March 21, 1996.

1991 Mathematics Subject Classification. Primary 46 L05.

Key words and phrases. Strictly positive element, hereditary $C *$-subalgebra, $Q$-commuting algebras, approximative units. 
In this version the result is certainly new, and we consider it interesting enough to merit attention. However, one of the corollaries in Brown's paper, [5, 3.35], shows how to deduce strict Urysohn results from ordinary ones. Although this method could be applied to the Akemann-Urysohn theorem, we believe that our approach is less involved, and - more importantly - the formulation is more user-friendly. The inspiration for the present work was a question raised by Loring (answered here as Corollary 4) about a detail in one of the arguments in [7] (Theorem 16.1.1), later also used in $[6,6.1]$.

Recall that if $A$ is a $C^{*}$-algebra with enveloping von Neumann algebra $A^{* *}$, a projection $p$ in $A^{* *}$ is open if it supports a hereditary $C^{*}$-subalgebra her $(p)$ of $A$, in which case

$$
p \in \operatorname{her}(p)^{-w} \quad \text { and } \quad \operatorname{her}(p)=p A^{* *} p \cap A,
$$

cf. $[8,3.10 .7,3.11 .10]$. If two open projections commute, we say that the corresponding hereditary $C^{*}$-subalgebras $q$-commute. The convergence condition in Theorem 1 is an equivalent formulation of $q$-commutativity (in the separable case), since every approximate unit for her $(p)$ converges strongly to $p$ in $A^{* *}$. A projection $p$ in $A^{* *}$ is closed if $1-p$ is open. Moreover, $p$ is compact if it is closed and dominated by some element in $A_{+}$, [3]. A complete discussion is found in [5, 2.D] (pp. 902-908). Finally, let us agree that an open projection $p$ is $\sigma$-compact if there is a sequence $\left(a_{n}\right)$ in $A_{+}$, such that $a_{n} \nearrow p$ (strong and monotone convergence in $\left.A^{* *}\right)$, i.e. if the associated hereditary $C^{*}$-subalgebra her $(p)$ is $\sigma$-unital. By $[5,3.34]$ $p$ is $\sigma$-compact if and only if it is the union of a sequence of compact projections. Evidently, these last notions are only interesting in the non-unital case, where we let $\widetilde{A}=A \oplus \mathbb{C}$ denote the unitized $C^{*}$-algebra obtained from $A$ (the minimal unitization) and denote by $M(A)$ the multiplier algebra of $A$ (the maximal unitization). Also the notion (by Aarnes and Kadison, cf. [8, 3.10.4]) of a strictly positive element $h$ in a $C^{*}$-algebra $A$ belongs to the non-unital theory. By definition $\varphi(h)>0$ for every state of $\varphi$ of $A$, which means that zero is not an eigenvalue for $h$ (in $\left.A^{* *}\right)$. Equivalently - and this is the main application - the sequence $\left(f_{n}(h)\right)$ is an approximate unit for $A$ for any choice of positive, continuous functions $\left(f_{n}\right)$ that vanish at zero and increase pointwise to 1 on $\operatorname{sp}(h) \backslash\{0\}$. Thus, whenever $A$ is $\sigma$-unital (i.e. has a countable approximate unit) it has a strictly positive element and thus an approximate unit consisting of pairwise commuting elements. Going further, such an approximate unit can even be chosen to commute approximately (in norm) with any given separable subset of $A$, cf. [8, 3.12.14].

Theorem 2. If $p$ is a compact and $q$ an open projection relative to a $C^{*}$-algebra $A$, such that $p \leq q$ and such that the open projection $q-p$ is $\sigma$-compact, then there is an element $h$ in $A$, with $p \leq h \leq q$, such that $h$ is strictly positive in $\operatorname{her}(q)$ and $1-h$ is strictly positive in $\operatorname{her}(1-p)$ (calculated in $\widetilde{A})$. Moreover, $h-h^{2}$ is strictly positive in her $(q-p)$.

Proof. The existence of an element $k$ in $A_{+}$with $p \leq k \leq q$ is the Akemann-Urysohn result as formulated e.g. in $[4,2.7]$. To obtain a strictly better element, choose a strictly positive element $b$ in $\operatorname{her}(q-p)$ with $\|b\|<1$. Then define

$$
h=(1-k)^{1 / 2} b(1-k)^{1 / 2}+k^{1 / 2}(1-b) k^{1 / 2}
$$


(cf. the formula $(*)$ from the introduction). Note that - working in $\widetilde{A}$ in the non-unital case -

$$
1-h=(1-k)^{1 / 2}(1-b)(1-k)^{1 / 2}+k^{1 / 2} b k^{1 / 2},
$$

so that $0 \leq h \leq 1$.

From the definition we see that

$$
h \leq(1-k)^{1 / 2} q(1-k)^{1 / 2}+k=(1-k) q+k q=q .
$$

Similarly,

$$
1-h \leq 1-k+k^{1 / 2}(1-p) k^{1 / 2}=1-p .
$$

It follows that $h \in \operatorname{her}(q)$ and $1-h \in \operatorname{her}(1-p)$ (inside $\widetilde{A})$. Note here that since $p$ is compact, $1-p$ is an open projection in $\widetilde{A}^{* *}$ (cf. [5, 2.47(iii)]), so that her $(1-p)$ is a hereditary $C^{*}$-subalgebra of $\widetilde{A}$. That $h-h^{2} \in \operatorname{her}(q-p)$ follows by a similar computation, or by using the fact that

$$
\operatorname{her}(q) \cap \operatorname{her}(1-p)=\operatorname{her}(q-p),
$$

since the open projections $q$ and $1-p$ commute.

To verify that $h$ is strictly positive in $\operatorname{her}(q)$, let $\varphi$ be a state of her $(q)$ and let $\varphi$ also denote its (unique) extension to a state of $\widetilde{A}$. Assume, to obtain a contradiction, that $\varphi(h)=0$. This implies that $\varphi(p)=0$ and moreover

$$
\varphi\left((1-k)^{1 / 2} b(1-k)^{1 / 2}\right)=0=\varphi\left(k^{1 / 2}(1-b) k^{1 / 2}\right) .
$$

Since $k$, and therefore also $(1-k)^{1 / 2}$, multiply her $(q-p)$, and since $\varphi$ is really a state of her $(q-p)$, it follows that $\varphi\left((1-k)^{1 / 2} \cdot(1-k)^{1 / 2}\right)$ is a positive functional on her $(q-p)$. As it annihilates the strictly positive element $b$ by $(* *)$, it must be zero, i.e. $\varphi(1-k)=0$. Thus $\varphi$ is supported by the spectral projection of $k$ corresponding to $\{1\}$, so $\varphi=\varphi\left(k^{1 / 2} \cdot k^{1 / 2}\right)$. In particular, by $(* *), \varphi(1-b)=0$; whence $\varphi(b)=1$, in contradiction with $\|b\|<1$.

The proof that $1-h$ is strictly positive in her $(1-p)$ is quite analogous: If $\varphi$ is a state of her $(1-p)$ that annihilates $1-h$, then necessarily $\varphi(1-q)=0$. Thus $\varphi(1-p)=1=\varphi(q)$, and since the open projections commute we conclude that $\varphi(q-p)=1$, so that $\varphi$ is a state of $\operatorname{her}(q-p)$. Now the contradiction follows as before, replacing $k$ by $1-k$ in the argument above.

To say that an element $a$ is strictly positive for a hereditary $C^{*}$-subalgebra $\operatorname{her}(z)$ of $A$ means exactly that the range projection $[a]$ of $a$ (i.e. the spectral projection of $a$ in $A^{* *}$, corresponding to the interval $] 0, \infty[$ ) is equal to $z$. Since we have shown that $[h]=q$ and $[1-h]=1-p$ it follows from spectral theory that

$$
\left[h-h^{2}\right]=[h][1-h]=q(1-p)=q-p .
$$

Thus $h-h^{2}$ is strictly positive for $\operatorname{her}(q-p)$.

Corollary 3. Let $A$ be a $C^{*}$-algebra and $B, C$ hereditary, q-commuting $C^{*}$-subalgebras of $A$ and $\widetilde{A}$, respecitively, such that $1 \in B+C$ and $B \cap C$ is $\sigma$-unital. There is then an element $h$ in $A$, with $0 \leq h \leq 1$, such that $h$ is strictly positive in $B$ and $1-h$ is strictly positive in $C$. Moreover, $h-h^{2}$ is strictly positive in $B \cap C$.

Proof. With $B=\operatorname{her}(q)$ and $C=\operatorname{her}(1-p)$ this is simply a translation of Theorem 2.

Note that Theorem 1 is the separable and unital case of this corollary. 
Corollary 4. Let $I$ be a $\sigma$-unital ideal in a $C^{*}$-algebra $A$. There is then for each projection $p$ in $A / I$ an element $h$ in $A$, with $0 \leq h \leq 1$, such that $h+I=p$ and $h-h^{2}$ is strictly positive in $I$.

Proof. Let $\pi: \widetilde{A} \rightarrow \widetilde{A} / I$ denote the quotient map and define

$$
\begin{aligned}
& B=\{x \in \widetilde{A} \mid \pi(x)(1-p)=(1-p) \pi(x)=0\}, \\
& C=\{x \in \widetilde{A} \mid \pi(x) p=p \pi(x)=0\} .
\end{aligned}
$$

Note that $B \subset A$, since we can find a lift $k$ of $p$ in $A$, whence $x \in B_{s a}$ if and only if $x(1-k) \in I$. Also, $B$ and $C$ are hereditary $C^{*}$-subalgebras with support projections $q+p$ and $1-p$, where $q$ denotes the open, central, $\sigma$-compact projection supporting $I$, and $p$ is identified with the compact projection $k(1-q)$. In particular, $B$ and $C$ are $q$-commuting. Evidently $1 \in B+C$ (as $k \in B$ and $1-k \in C$ ) and $B \cap C=I$, so the previous corollary applies.

\section{REFERENCES}

[1] C.A. Akemann, The general Stone-Weierstrass problem, J. Funct. Anal. 4 (1969), 277-294. MR 40: 4772

[2] C.A. Akemann, Left ideal structure of $C^{*}$-algebras, J. Funct. Anal. 6 (1970), 305-317. MR 43:934

[3] C.A. Akemann, A Gelfand representation theory for $C^{*}$-algebras, Pacific J. Math. 39 (1971), 1-11. MR 48:6950

[4] C.A. Akemann and G.K. Pedersen, Facial structure in operator algebra theory, Proc. London Math. Soc. (3) 64 (1992), 418-448. MR 93c:46016

[5] L.G. Brown, Semicontinuity and multipliers of $C^{*}$-algebras, Canad. J. Math. 40 (1988), 865-988. MR 90a:46148

[6] S. Eilers, T.A. Loring and G.K. Pedersen, Morphisms of extensions of $C^{*}$-algebras: Pushing forward the Busby invariant, Advances in Math., to appear.

[7] T.A. Loring, "Lifting Solutions to Perturbing Problems in $C^{*}$-Algebras", Fields Institute Monographs 8, Amer. Math. Soc., Providence, 1997.

[8] G.K. Pedersen, "C*-Algebras and their Automorphism Groups", LMS monographs 14, Academic Press, London, 1979. MR 81e:46037

Mathematics Institute, University of Copenhagen, Universitetsparken 5, DK-2100, Copenhagen $\varnothing$, Denmark

E-mail address: gkped@math.ku.dk 